University of Nebraska - Lincoln

DigitalCommons@University of Nebraska - Lincoln

John R. Hardy Papers

Research Papers in Physics and Astronomy

7-15-1968

Infrared Absorption Properties of LiH, LiD Mixed Crystals

Sitaram S. Jaswal

University of Nebraska - Lincoln, sjaswal1@unl.edu

John R. Hardy

University of Nebraska - Lincoln

Follow this and additional works at: https://digitalcommons.unl.edu/physicshardy

Part of the Physics Commons

Jaswal, Sitaram S. and Hardy, John R., "Infrared Absorption Properties of LiH, LiD Mixed Crystals" (1968). John R. Hardy Papers. 9.

https://digitalcommons.unl.edu/physicshardy/9

This Article is brought to you for free and open access by the Research Papers in Physics and Astronomy at DigitalCommons@University of Nebraska - Lincoln. It has been accepted for inclusion in John R. Hardy Papers by an authorized administrator of DigitalCommons@University of Nebraska - Lincoln. 


\title{
Infrared Absorption Properties of LiH, LiD Mixed Crystals*
}

\author{
S. S. JASWAL \\ Behlen Laboratory of Physics, University of Nebraska, Lincoln, Nebraska \\ AND \\ J. R. HARDY $\dagger$ \\ Lawrence Radiation Laboratory, University of California, Livermore, California
}

\begin{abstract}
The normal modes of pure $\mathrm{LiH}$ and $\mathrm{LiD}$, required in impurity mode calculations, have been computed on the basis of the deformation-dipole model. Neutron-scattering results of Verble, Warren, and Yarnell for $\mathrm{Li}^{7} \mathrm{D}$, along with some other experimental quantities, have been used to determine the various parameters involved. It is found that the frequency spectrum of $\mathrm{LiH}$ has a gap and that a substitutional $\mathrm{D}^{-}$ion produces a local mode in this gap whose frequency has been computed, together with its amplitude at the impurity. The infrared absorption frequency associated with this local mode and its integrated absorption have been computed as functions of $\mathrm{D}^{-}$content in the limit of low $\mathrm{D}^{-}$concentration. Also, we find an in-band resonance in the amplitude of the substitutional $\mathrm{D}^{-}$ion. Similar calculations have been carried out for $\mathrm{H}^{-}$impurities in LiD. The present results are compared with those of other calculations and with experiment.
\end{abstract}

\section{INTRODUCTION}

$\mathrm{F}$ OR the purpose of studying impurity effects on lattice vibrations, mixed LiH-LiD systems are ideal, since they are true isotopic mixtures and the $\mathrm{H}$, $\mathrm{D}$ mass difference is large enough to produce marked changes in the vibrational properties of these systems as the relative concentrations of the two components are varied. Some time ago, with this fact in mind, Misho ${ }^{1}$ carried out infrared absorption measurements on thin films of such mixtures. He found that the addition of a small amount of $\mathrm{D}^{-}$to $\mathrm{LiH}$ produces a large decrease in the main absorption frequency below the fundamental infrared dispersion frequency $\left(\omega_{\mathrm{To}}\right)$ of pure LiH.

Our main object in the present paper is to attempt to understand Misho's results theoretically, but, in order to do this, we first need an adequate theoretical treatment of the dynamics of both pure $\mathrm{LiH}$ and pure $\mathrm{LiD}$.

Previously this has not been possible because of lack of knowledge of the necessary input parameters, e.g., experimental values ${ }^{2,3}$ of the compressibility vary over a wide range. Recently, Benedek ${ }^{4}$ reported some calculations on the lattice dynamics of $\mathrm{LiH}$ and $\mathrm{LiD}$ using the deformation-dipole (DD) model $^{5}$ but we believe that these are in error, since for both the alternative sets of input data he uses, we find that the lattice is unstable against certain normal-mode displacements within the reduced zone.

* This work was performed under the auspices of the U. S. Atomic Energy Commission.

$\dagger$ Permanent address: Behlen Laboratory of Physics, University of Nebraska, Lincoln, Neb.

${ }^{1}$ R. H. Misho, Ph.D. thesis, Michigan State University, 1961 (unpublished).

${ }^{2}$ F. E. Pretzel, G. N. Rupert, C. L. Mader, E. K. Sturms, G. V. Gritton, and C. C. Rushing, J. Phys. Chem. Solids 16, 10 (1960).

${ }^{3}$ R. Weil and A. W. Lawson, J. Chem. Phys. 37, 2730 (1962);

F. F. Voronov, V. A. Goncharova, O. V. Slal'gorova, and T. A. Agapova, Fiz. Tverd. Tela 8, 1643 (1966) [English transl.: Soviet Phys.-Solid State 8, 1313 (1966)].

${ }^{4}$ G. Benedek, Solid State Commun. 5, 101 (1967).

${ }^{5}$ J. R. Hardy, Phil. Mag. 7, 315 (1962).
For example, for a compressibility $\beta=2.55 \times 10^{-12}$ $\mathrm{cm}^{2} /$ dyn and a $\mathrm{H}^{-}$polarizability $\alpha^{-}=1.9 \times 10^{-24}$, some of the frequencies are imaginary for reduced wave vectors q equal to $(0.2,0.2,0),(0.3,0.3,0.1),(0.4,0.4,0)$, $(0.4,0.4,0.2),(0.4,0.4,0.4),(0.5,0.5,0.3)$, and $(0.5,0.5$, $0.5)$. Also, his expression for $\omega_{\text {To }}$ appears to be incorrect. When the short-range interactions are approximated by central forces between nearest neighbors only, the correct expression for $\omega_{\text {TO }}$ is

$\omega_{\mathrm{TO}}^{2}=\frac{1}{\bar{M}} \frac{e^{2}}{v_{a}}\left[A+2 B-\frac{4}{3} \pi s^{2} \frac{1}{1-\left(4 \pi / 3 v_{a}\right)\left(\alpha^{+}+\alpha^{-}\right)}\right]$,

where $v_{a}$ is the volume of a primitive cell, $e$ is the electronic charge, $\bar{M}$ is the reduced mass of the positive and negative ions, $A$ and $B$ are the short-range force constants defined by Kellermann, ${ }^{6} s$ is the ratio of the Szigeti charge ${ }^{7}$ to the electronic charge, and $\alpha^{+}$and $\alpha^{-}$ are, respectively, the positive- and negative-ion crystal polarizabilities.

Montgomery and Hardy ${ }^{8}$ explained the experimental results of Misho in terms of a local mode in a gap in the $\mathrm{LiH}$ frequency spectrum. They approximated the $\mathrm{LiH}$ lattice by a monatomic face-centered cubic lattice made up of $\mathrm{H}^{-}$ions, and to estimate the frequency spectrum they used the neutron scattering data of Woods $e t a l .^{9}$ which give only the optical modes. More recently, Elliott and Taylor ${ }^{10}$ made a more elaborate study of the $\mathrm{LiH}: \mathrm{D}$ and $\mathrm{LiD}: \mathrm{H}$ systems and calculated the local mode frequency and the integrated local mode absorption as functions of defect concentration. In this case, the optical mode frequency distribution of $\mathrm{LiH}$

${ }^{6}$ E. W. Kellermann, Phil. Trans. Roy. Soc. (London) A238, 513 (1940)

${ }_{7}$ B. Szigeti, Proc. Roy. Soc. (London) A204, 51 (1950).

8 D. J. Montgomery and J. R. Hardy, J. Phys. Chem. Solids, Suppl. 1, 491 (1965).

${ }^{9}$ A. D. B. Woods, B. N. Brockhouse, M. Sakomoto, and R. N. Sinclair, in Inelastic Scattering of Neutrons in Solids and Liquids (International Atomic Energy Agency, Vienna, 1961), p. 487.

${ }^{10}$ R. J. Elliott and D. W. Taylor, Proc. Roy. Soc. (London) A296, 161 (1967).

1090 
was approximated by fitting two Gaussians to the experimental results of Woods et al. ${ }^{9}$ Evidently, both theories are limited by lack of complete knowledge of the frequency spectrum of the perfect crystal.

Recently, Verble et al. ${ }^{11}$ have obtained by neutron scattering most of the dispersion curves for $\mathrm{Li}^{7} \mathrm{D}$ along three symmetry directions. In the present work, we have computed the normal modes of $\mathrm{LiH}$ and $\mathrm{LiD}$ on the basis of the DD model and, in order to determine the various parameters involved, we have used these results along with other experimental quantities. With the help of the calculated eigenfrequencies and the eigenvectors of the pure crystals, we have carried out impurity-mode calculations for both $\mathrm{LiH}: \mathrm{D}$ and $\mathrm{LiD}: \mathrm{H}$. The results of the present calculations are compared with the experimental data of Misho, ${ }^{1}$ and Elliott and Taylor's ${ }^{10}$ theoretical work. A preliminary report on the impurity-mode calculations in $\mathrm{LiH}: \mathrm{D}$ has been given elsewhere. ${ }^{12}$

\section{LATTICE DYNAMICS OF THE PURE SYSTEMS}

We compute the normal modes of the pure systems on the basis of the DD model and within the harmonic approximation. The short-range interactions consist of central forces between nearest and next-nearest neighbors and an angle-bending force. ${ }^{13} \mathrm{LiH}$ has the $\mathrm{NaCl}$ structure, thus any two similar ions which are nearest neighbors of a dissimilar ion and lie along the positive directions of any two coordinate axes subtend a right angle at the dissimilar ion. The angle-bending force is proportional to the deviation from this right angle. The proportionality constant $C$ is assumed to be the same with either kind of ion at the origin. In this approximation the contributions of the angle-bending force to various elements of the dynamical matrix $D$ are as follows:

$$
\begin{aligned}
D_{x x}{ }^{B}\left(K K^{\prime}: \mathbf{q}\right) & =-\left(M_{K} M_{K^{\prime}}\right)^{-1 / 2}\left(e^{2} / v_{a}\right) 4 C \\
\times\left(\cos \pi q_{y}+\cos \pi q_{z}\right) & \\
D_{x y} B\left(K K^{\prime}: \mathbf{q}\right) & =0, \\
D_{x x} B(K K: \mathbf{q}) & =\left(1 / M_{K}\right)\left(e^{2} / v_{a}\right) 8 C, \\
D_{x y} B(K K: \mathbf{q}) & =\left(1 / M_{K}\right)\left(e^{2} / v_{a}\right) 2 C \sin \pi q_{x} \sin \pi q_{y},
\end{aligned}
$$

where $K$ and $K^{\prime}$ label the two kinds of ions, $\mathbf{q}$ is the reduced wave vector, and $C$ is the angle-bending force constant. These expressions are symmetric in the interchange of $x, y$, etc., and $K$ and $K^{\prime}$.

The next-nearest-neighbor (NNN) forces between positive-positive and negative-negative ions are not

${ }^{11}$ J. L. Verble, J. L. Warren, and J. L. Yarnell, Bull. Am. Phys. Soc. 12, 557 (1957). (We should also like to thank these authors for private communication of a fuller account of this work.)

${ }^{12} \mathrm{~S}$. S. Jaswal and J. R. Hardy, in Proceedings of the Irvine Conference on Localized Excitations in Solids (to be published).

${ }_{13}$ A. A. Maradudin (private communication). We should like to thank Professor Maradudin for access to this unpublished work.

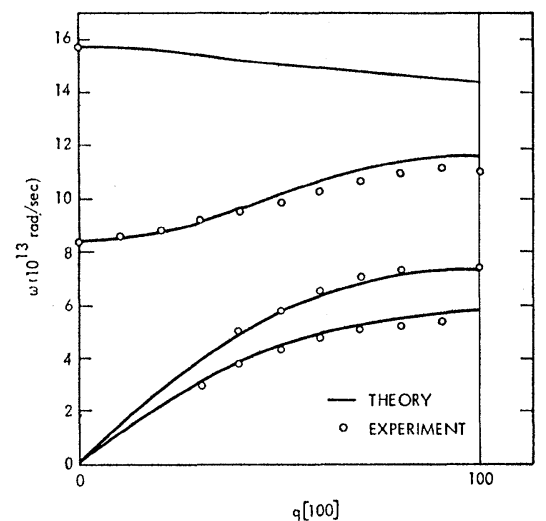

(a)

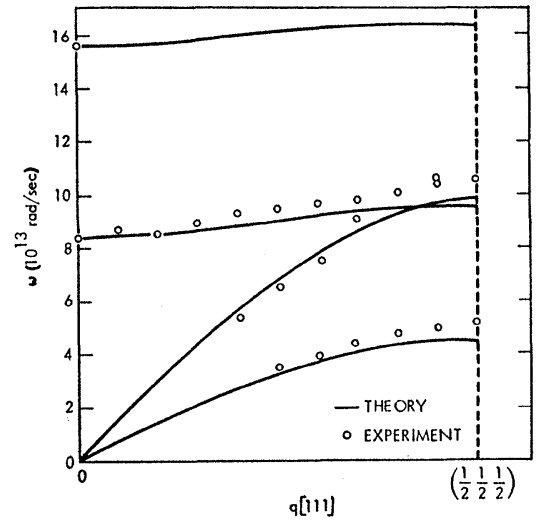

(b)

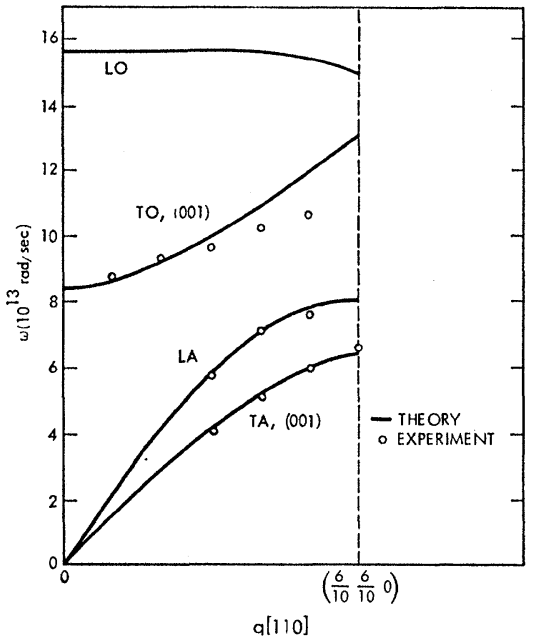

(c)

FIG. 1. Phonon-dispersion curves in the three symmetry directions for $\mathrm{Li}^{7} \mathrm{D}$.

treated independently, since their ratio is assumed to be a constant which is used as an adjustable parameter.

The monopole charge $Z$ is allowed to vary and the positive and negative ion polarizabilities are adjusted so that their sum is equal to $1.893 \times 10^{-24} \mathrm{~cm}^{-3}$, the value obtained from the Clausius-Mossotti relation. 


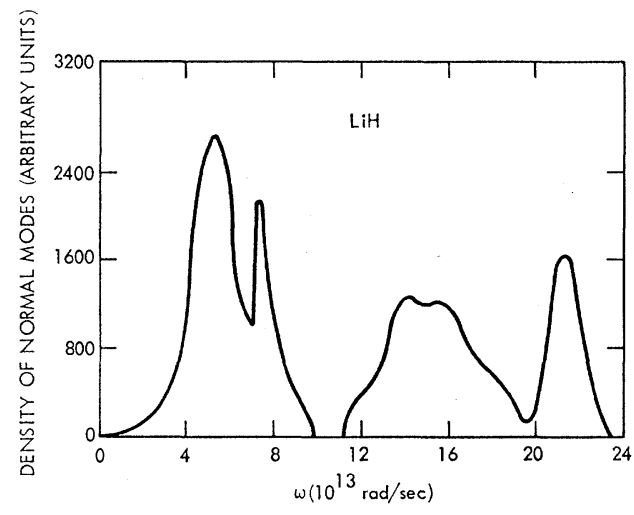

FIG. 2. Frequency distribution for $\mathrm{LiH}$.

To adjust the various parameters involved, we first computed the normal modes of $\mathrm{Li}^{7} \mathrm{D}$. In addition to using the measured phonon dispersion curves of Verble et $a l .{ }^{11}$ as a guide in our calculations, we also used the lattice parameter $r_{0},{ }^{2}$ the infrared dispersion frequency $\omega_{\mathrm{TO}},{ }^{14}$ the Szigeti charge $s=0.52,{ }^{14}$ and the elastic constants $C_{11}$ and $C_{44}$ derived from the longitudinal acoustic and transverse acoustic branches of the experimental phonon dispersion curves of $\mathrm{Li}^{7} \mathrm{D}$ in the symmetry direction [100].

Thus with elastic constants

$$
\begin{aligned}
& C_{11}=6.63 \times 10^{11} \mathrm{dyn} / \mathrm{cm}^{2}, \\
& C_{44}=4.83 \times 10^{11} \mathrm{dyn} / \mathrm{cm}^{2},
\end{aligned}
$$

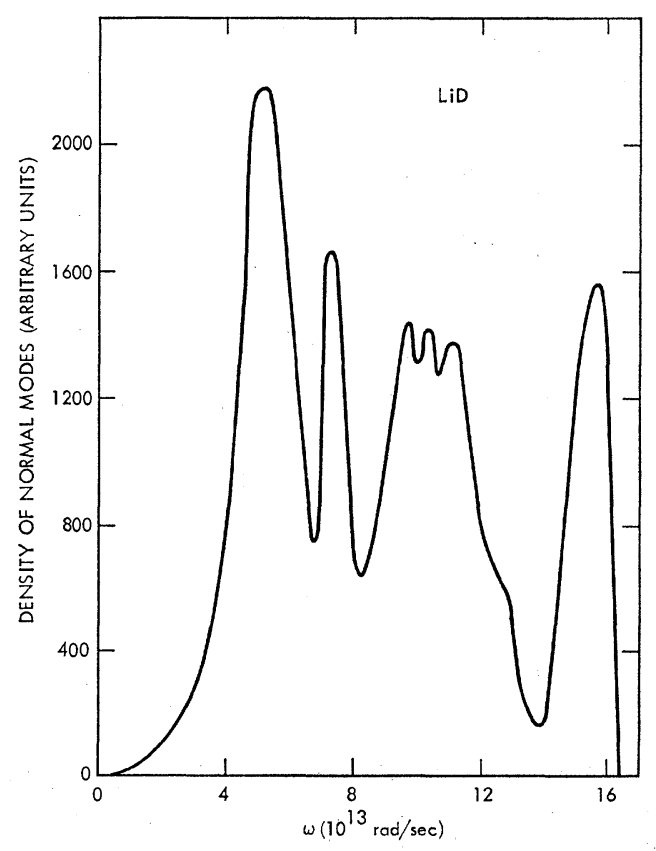

FIG. 3. Frequency distribution for $\mathrm{LiD}$.

${ }^{14}$ D. J. Montgomery and K. F. Yeung, J. Chem. Phys. 37, 1056 (1962). the best fit was given by $C \times\left(e^{2} / 2 r_{0}{ }^{4}\right)=0.841 \times 10^{11} \mathrm{cgs}$ units; $A_{1}=3.85, B_{1}=-0.69, A_{2}{ }^{++}=0.24, A_{2}^{--}=0.36$, $B_{2}{ }^{++}=0.025, B_{2}^{--}=0.037$ (all in Kellermann's units ${ }^{6}$; the subscripts 1 and 2 refer to $\mathrm{NN}$ and NNN, respectively) $|Z|=0.7|e|$.

$$
\frac{\text { NNN potential for positive ions }}{\text { NNN potential for negative ions }}=\frac{2}{3},
$$

$$
\alpha^{+} \text {(positive ion polarizability) }=0.229 \times 10^{-24} \mathrm{~cm}^{-3},
$$$$
\alpha^{-} \text {(negative ion polarizability) }=1.664 \times 10^{-24} \mathrm{~cm}^{-3} \text {. }
$$

Our computed phonon dispersion curves in the three symmetry directions are compared with the experimental results in Fig. 1. In all three cases, the agreement between theory and experiment at low $\mathbf{q}$ is very good, but the deviation increases toward the zone boundary. However, except for the transverse optical branch at the zone face along the [110] direction and the transverse acoustic branch at the zone face along the [111] direction, where the disagreement is $\sim 13 \%$, this deviation is about $6 \%$ or less. Thus the overall agreement between the calculated and the experimental results is satisfactory from the point of view of defect calculations. We would like to remark that our longitudinal optical frequencies are consistently higher than those of Verble $e t a l .^{11}$ obtained from a shell model.

As regards the adjustable parameters given above, we think they are quite realistic. The value of monopole charge is less than the free-electron charge, since lithium hydride is not as ionic as the alkali halides, ${ }^{2}$ while the ratio of the NNN potentials of the two ions is comparable with that of their ionic radii. While it is probable that we could have improved the agreement between calculated and experimental dispersion curves by least-squares fitting the resultant model parameters would be less realistic (e.g., the high-frequency dielectric constant would be in error) and we have chosen to obtain the best fit we can without varying the input parameters beyond reasonable limits.

The parameters obtained for $\operatorname{Li}^{7} \mathrm{D}$ are then used to compute the normal modes of $\mathrm{LiH}$ and $\mathrm{LiD}$. The experimental values of $\omega_{\text {To }}$ and $s$ for these systems are

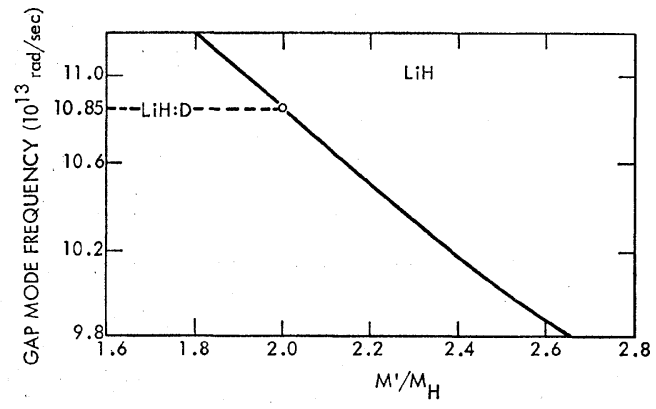

FIG. 4. Gap-mode frequency in $\mathrm{LiH}$ as a function of impurity mass $M^{\prime}$. 


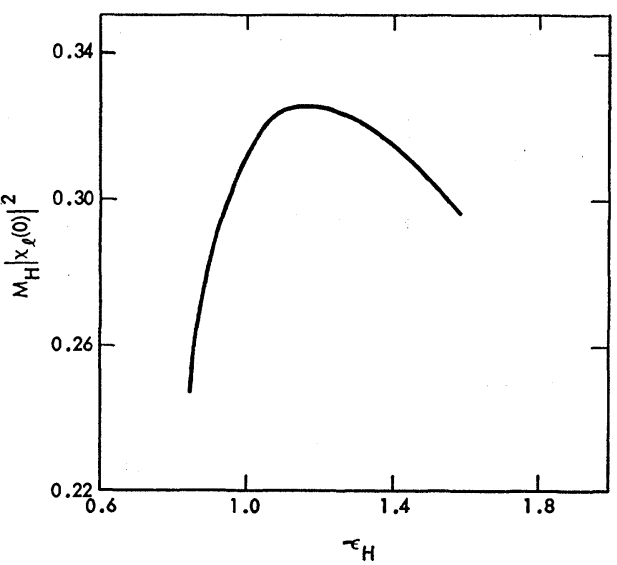

FIG. 5. Amplitude of vibration of the impurity atom in $\mathrm{LiH}$ as a function of $\epsilon_{\mathrm{H}}$.

those due to Zimmerman and Montgomery ${ }^{15}$ and Brodsky. ${ }^{16}$ Our computed frequency distribution for $\mathrm{LiH}$ is shown in Fig. 2. Our longitudinal optical peak is at a somewhat higher frequency than that obtained by incoherent neutron scattering by Woods et al..$^{9} \mathrm{It}$ can be seen that there is a gap in the frequency spectrum of $\mathrm{LiH}$, bounded by the frequencies $9.83 \times 10^{13}$ and $11.2 \times 10^{13} \mathrm{rad} / \mathrm{sec}$. From the phonon dispersion curves in Fig. 1, it is clear that the upper edge of the gap is quite accurate, whereas the lower edge is uncertain by about $10 \%$. However, since the local mode we are concerned with is near the upper edge of the gap, our computed frequency spectrum should be adequate to study its properties. Figure 3 shows the computed frequency distribution of $\mathrm{LiD}$ which has no gap, a fact consistent with the experimental results of Verble et al. ${ }^{11}$ Both frequency distributions have been derived from samples of 8000 points within the first Brillouin zone.

\section{IMPURITY MODES IN LiH:D}

On the basis of these results, we have studied the impurity modes in $\mathrm{LiH}$ containing low concentrations of deuterium. Since $\mathrm{LiH}$ has a gap in its frequency spectrum, heavier impurities substituted for $\mathrm{H}^{-}$will, in general, give rise to localized modes in this gap. For such substitutional mass defects, the solution of the standard eigenvalue equation ${ }^{17}$ gives the local-mode frequency as a function of the relative change in mass. The results for gap modes due to heavier impurities substituted for an $\mathrm{H}^{-}$ion in $\mathrm{LiH}$ are shown in Fig. 4, where $M^{\prime}$ is the mass of the impurity. From this we find that a substitutional deuterium gives rise to a local

${ }^{15}$ W. B. Zimmerman and D. J. Montgomery, Phys. Rev. 120, 405 (1960).

${ }_{16}$ M. Brodsky, Ph.D. thesis, University of Pennsylvania, 1965 (unpublished).

17 A. A. Maradudin, in 1962 Brandeis University Summer Institute Lectures in Theoretical Physics (W. A. Benjamin, Inc., New York, 1962), Vol. II.

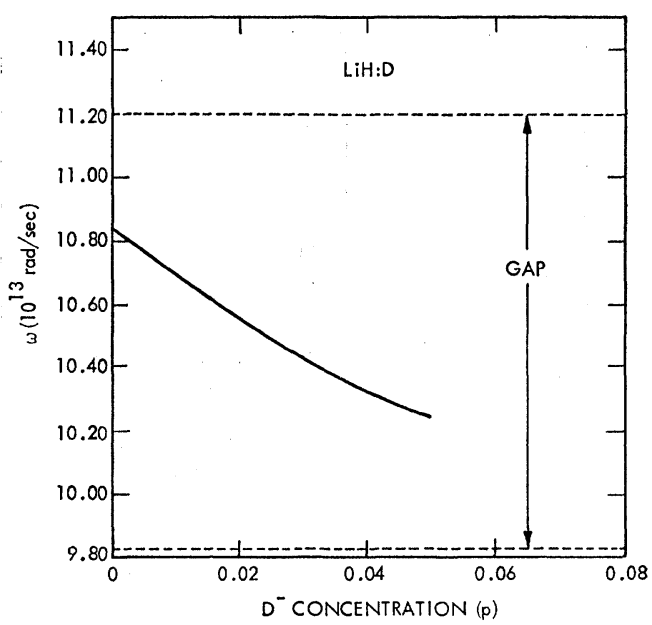

FiG. 6. Absorption frequency as a function of $\mathrm{D}^{-}$concentration in $\mathrm{LiH}$.

mode in the gap at a frequency $\omega_{1}=10.85 \times 10^{13} \mathrm{rad} / \mathrm{sec}$, which is close to $11.21 \times 10^{13} \mathrm{rad} / \mathrm{sec}$, the value of $\omega_{\text {To }}$ for pure $\mathrm{LiH}$.

Also, we have computed the amplitude ${ }^{18}\left|\chi_{1}(0)\right|^{2}$ of the impurity atom in these local modes and the results are shown in Fig. 5, where $\epsilon_{\mathrm{H}}=\left(M_{\mathrm{H}}-M^{\prime}\right) / M_{\mathrm{H}}$, $M_{\mathrm{H}}$ being the mass of $\mathrm{H}^{-}$. We see that the amplitude as a function of $\epsilon_{\mathrm{H}}$ goes through a maximum, whereas the local mode frequency decreases monotonically as the mass of impurity atom increases. For a deuterium impurity, $M_{\mathrm{H}}\left|\chi_{1}(0)\right|^{2}=0.312$.

Since these local modes are optically active, infrared spectroscopy can be used to study them experimentally. However, the infrared absorption frequency shifts considerably with an increase in defect concentration $p$. Various workers have studied this problem for low defect concentrations. ${ }^{17,10,19}$ According to Maradudin, ${ }^{17}$ the following equation gives the absorption frequency as a function of $p$ :

$$
\omega_{\mathrm{TO}}{ }^{2}-\omega^{2}+\epsilon_{\mathrm{H}} \mu p \omega_{\mathrm{TO}}{ }^{2} A^{-1}(\omega)=0,
$$

where $\omega$ is the frequency to be determined, $\mu=M_{\mathrm{Li}}$ /

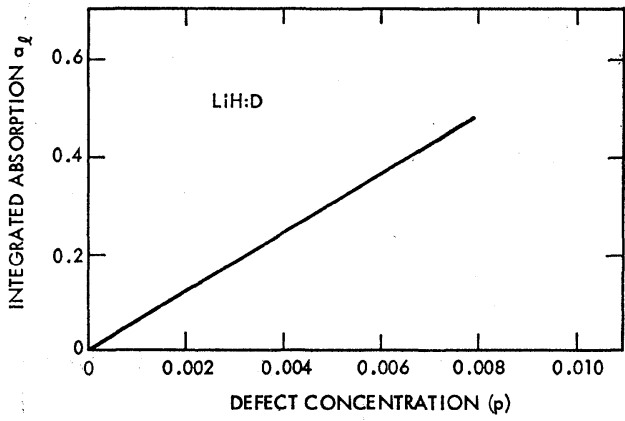

Fig. 7. Integrated absorption in the gap mode as a function of $\mathrm{D}^{-}$concentration in $\mathrm{LiH}$.

${ }^{18}$ S. S. Jaswal, J. Chem. Phys. 46, 4311 (1967)

${ }^{19}$ G. Benedek and G. F. Nardelli, Phys. Rev. 155, 1004 (1967). 


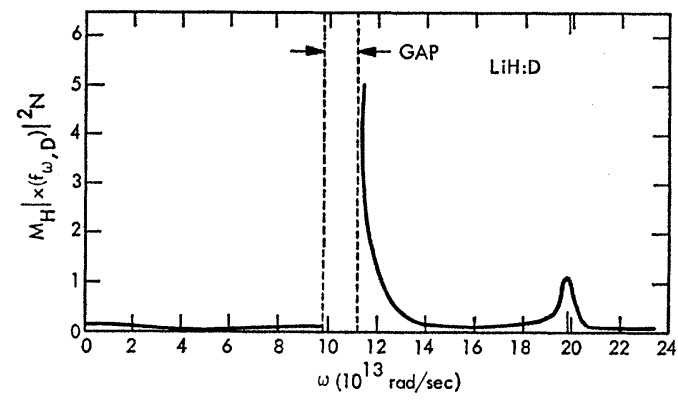

FIG. 8. Amplitude of vibration of a $\mathrm{D}^{-}$ion in $\mathrm{LiH}$ as a function of band-mode frequency.

$\left(M_{\mathrm{Li}}+M_{\mathrm{H}}\right)$, and

$$
A(\omega)=1-\frac{\epsilon_{\mathrm{H}} \omega^{2}}{3 N} \sum_{\mathbf{q} j} \frac{|\mathbf{W}(-\mid q, j)|^{2}}{\omega^{2}-\omega_{j}^{2}(\mathbf{q})}
$$

where $N$ is the number of unit cells in the crystal, $W(-\mid q, j)$ is the negative-ion eigenvector amplitude, and $\omega_{j}(\mathbf{q})$ is the eigenfrequency corresponding to wave vector $\mathbf{q}$ and branch index $j$.

We have solved Eq. (3.1) numerically for various concentrations of $\mathrm{D}^{-}$impurities in $\mathrm{LiH}$, and the resultant $\omega$-versus- $p$ curve is shown in Fig. 6 . The absorption frequency decreases by about $6 \%$ as $p$ goes from 0 to $5 \%$. These results are in agreement with those of Elliott and Taylor' ${ }^{10}$ calculations, based on their approximate form of the $\mathrm{LiH}$ frequency spectrum.

The relative integrated absorption $a_{1}$ in the local mode, i.e., the ratio of the absorption in the local mode to that at the transverse optic frequency of the perfect crystal, is equal to the ratio of the squares of the electric dipole moments of these two modes. The local-mode dipole moment $\mu_{1}$ of the crystal is given by

$$
\mu_{1}=\sum_{K l} e_{K}{ }^{*} \chi(K \mid 1, l),
$$

where $e_{K}^{*}$ is an effective charge $\left(\left|e_{+}{ }^{*}\right|=\left|e_{-}^{*}\right|=e^{*}\right)$ and

$$
\begin{aligned}
& \chi(K \mid 1, l)=-\frac{1}{N} \sum_{\alpha K^{\prime} \mathbf{q} j} \frac{W^{*}(K \mid q, j) W_{\alpha}\left(K^{\prime} \mid q, j\right)}{\omega_{j}{ }^{2}(\mathbf{q})-\omega_{1}{ }^{2}} \\
& \times \chi_{\alpha}\left(K^{\prime} \mid 1,0\right) \epsilon_{K^{\prime}}\left(M_{K^{\prime}} / M_{K}\right)^{1 / 2} \omega_{1}{ }^{2} \exp \left(-\frac{i \pi}{r_{0}} \mathbf{q} \cdot \mathbf{R}_{l}\right)
\end{aligned}
$$

is the amplitude ${ }^{20}$ of $K$ th ion in the $l$ th cell corresponding to the local mode 1 . When the various summations in (3.3) are carried out, the only terms which survive on the right-hand side are those corresponding to transverse optical modes at $\mathbf{q}=0$. The final result for the square of the dipole moment becomes

$$
\begin{array}{r}
\mu_{1}^{2}=e^{* 2} \mu \epsilon_{\mathrm{H}}{ }^{2} \frac{\omega_{1}^{4}}{\left(\omega_{\mathrm{TO}}{ }^{2}-\omega_{1}^{2}\right)^{2}}\left|\frac{\mathrm{W}(+\mid 0, j)}{\sqrt{ } M_{+}}-\frac{\mathrm{W}(-\mid 0, j)}{\sqrt{ } M_{-}}\right|^{2} \\
\times M_{\mathrm{H}}\left|\chi_{1}(0)\right|^{2},
\end{array}
$$

${ }^{20}$ P. G. Dawber and R. J. Elliott, Proc. Roy. Soc. (London) A273, 222 (1963). where we have taken into account the threefold degeneracy of the local mode. Here $j$ refers to the transverse optical branch. The corresponding result for the transverse optic mode of the perfect crystal is

$$
\mu_{\mathrm{To}}{ }^{2}=e^{* 2} N\left|\frac{\mathrm{W}(+\mid 0, j)}{\sqrt{ } M_{+}}-\frac{\mathrm{W}(-\mid 0, j)}{\sqrt{ } M_{-}}\right|^{2} .
$$

Thus, the relative integrated absorption for a low concentration of defects $p$ is given by

$$
a_{1}=p \mu \frac{\epsilon_{\mathrm{H}^{2} \omega_{1}{ }^{4}}}{\left(\omega_{1}^{2}-\omega_{\mathrm{TO}}\right)^{2}} M_{\mathrm{H}}\left|\chi_{1}(0)\right|^{2} .
$$

This expression holds exactly only when the local modes centered on different defects do not interact, and $p$ must be very low for this to be true.

Using this expression, we have computed $a_{1}$ as a function of $p$ for $\mathrm{LiH}: \mathrm{D}$ and the results are shown in Fig. 7. First of all we see that at very low concentrations of $\mathrm{D}^{-}$, where our expression holds exactly, the absorption in the local mode forms a significant fraction of that in $\omega_{\text {To }}$. Secondly, even through our expression does not hold for higher defect concentrations, our results nonetheless indicate a rapid increase in the integrated absorption $a_{1}$ as $p$ is increased further, e.g., for $p$ as low as $0.01, a_{1} \sim 0.5$. Once again, our low concentration results are similar to those of Elliott and Taylor. ${ }^{10}$ Also, they are in qualitative agreement with experiment, ${ }^{1}$ i.e., the calculated integrated absorption of the so-called local mode becomes very large even for $p$ as low as 0.02 , but, even allowing for the shift with concentration, our computed absorption frequency is considerably higher than the experimental value, ${ }^{1}$ of $8.49 \times 10^{13} \mathrm{rad} / \mathrm{sec}$ for a crystal of $\mathrm{LiH}$ containing $5 \%$ $\mathrm{LiD}$.

To investigate the existence of in-band resonance modes associated with $\mathrm{D}^{-}$impurities in $\mathrm{LiH}$, we have computed the amplitude of the impurity atom as a

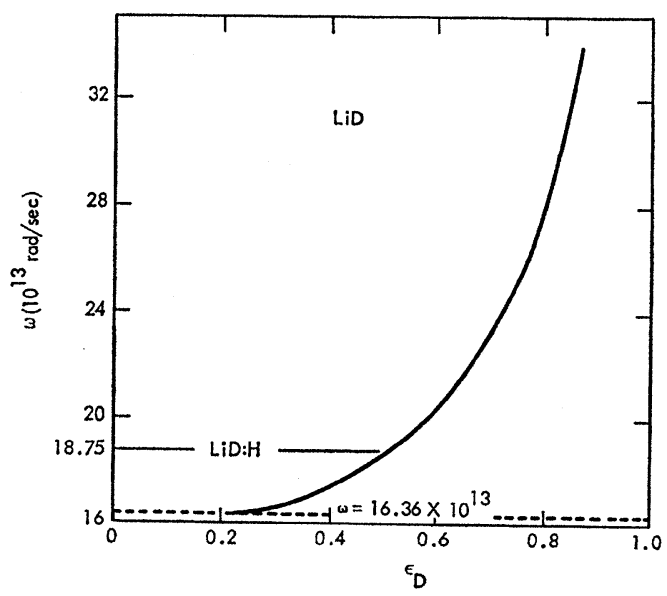

FIG. 9. Local-mode frequency in $\mathrm{LiD}$ as a function of $\epsilon_{\mathrm{D}}$. 

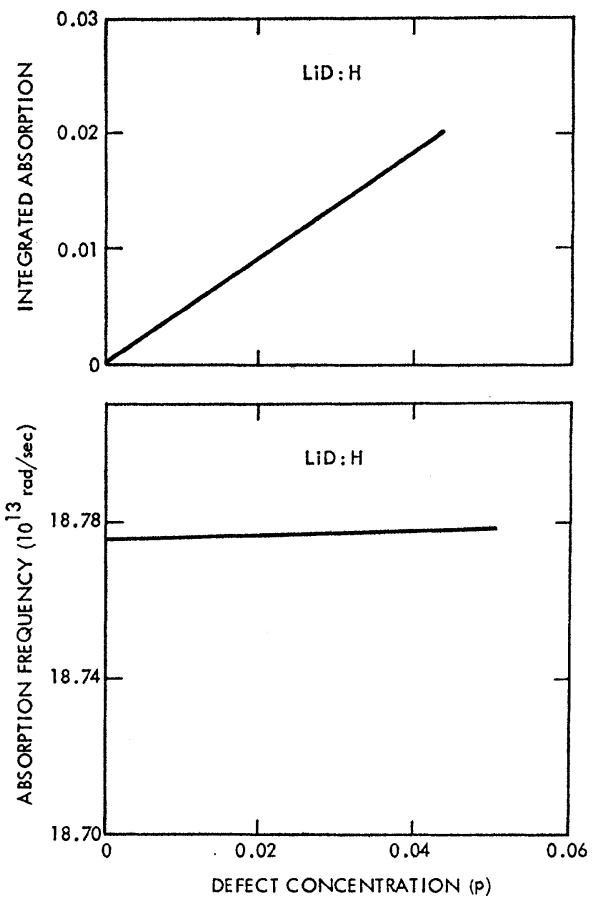

FIG. 10. Absorption frequency and integrated absorption in the local mode as functions of $\mathrm{H}^{-}$concentration in $\mathrm{LiD}$.

function of frequency within the ranges spanned by the spectrum of pure $\mathrm{LiH}$, and the results are shown in Fig. 8. The large increase in the amplitude near the upper edge of the gap is due to the nearness of the local mode, and we also predict a resonance in the optical part of the frequency spectrum at $19.8 \times 10^{13} \mathrm{rad} / \mathrm{sec}$.

\section{IMPURITY MODES IN LiD:H}

Some of the impurity-mode calculations of Sec. 3 have been repeated for $\mathrm{H}^{-}$impurities in LiD. Figure 9 shows the local mode frequency as a function of the mass defect $\epsilon_{D}$ when a lighter impurity is substituted for a $\mathrm{D}^{-}$ion. From this we see that a substitutional $\mathrm{H}^{-}$ ion produces a local mode at a frequency $\omega_{1}=18.75 \times 10^{13}$ $\mathrm{rad} / \mathrm{sec}$, which lies above the maximum frequency of the pure crystal. This value is about $4 \%$ higher than that obtained by Elliott and Taylor ${ }^{10}$ for the same system.

Plots of the infrared absorption frequency and the integrated absorption against defect concentration are shown in Fig. 10. The absorption frequency increases slightly with increase in $p$, contrary to the results of Elliott and Taylor. ${ }^{10}$ The integrated absorption in the local mode is quite small and increases linearly with $p$ in the approximation we are using. For example, the integrated absorption is about $2 \%$ for $p=0.05$, and this probably is the reason why Misho failed to see the local mode in his experiments on LiD:H. Moreover, our predictions with regard to the integrated intensity agree with those of Elliott and Taylor. ${ }^{10}$

\section{CONCLUSIONS}

We think that we now have a reasonably good representation of the normal modes of $\mathrm{LiH}$ and $\mathrm{LiD}$, and hence our impurity-mode calculations are quite realistic. A low concentration of $\mathrm{D}^{-}$in $\mathrm{LiH}$ gives a local mode in the gap whose frequency decreases with increase in defect concentration. The integrated absorption in the gap mode is quite large, even for low concentration of $\mathrm{D}^{-}$, but the absorption frequency is much higher than that measured experimentally by Misho. ${ }^{1}$ Also, we find that the substitutional $\mathrm{D}^{-}$has a resonance mode in the optical part of the frequency distribution of $\mathrm{LiH}$.

A substitutional $\mathrm{H}^{-}$in $\mathrm{LiD}$ gives rise to a local mode above the maximum frequency of the pure crystal whose frequency increases slightly with increase in $\mathrm{H}^{-}$concentration. The integrated absorption in the local mode is small and increases linearly with the defect concentration. This absorption has not so far been seen experimentally.

To resolve the discrepancy between the calculated and the observed local mode absorption frequencies for $\mathrm{D}^{-}$impurities in $\mathrm{LiH}$, more experimental work is desirable. Also, it should be possible to observe the local mode produced by $\mathrm{H}^{-}$impurities in $\mathrm{LiD}$.

Finally, it is obvious that the behavior of truly mixed crystals, containing comparable amounts of $\mathrm{H}^{-}$and $\mathrm{D}^{-}$, is very little understood either theoretically or experimentally. 